\title{
Aspectos clínicos e epidemiológicos da leishmaniose visceral em menores de 15 anos procedentes de Alagoas, Brasil
}

\author{
Clinical and epidemiological aspects of visceral leishmaniasis in \\ children up to 15 years of age in Alagoas, Brasil
}

Célia Maria Silva Pedrosa ${ }^{1,2}$ e Eliana Maria Mauricio da Rocha ${ }^{3}$

\begin{abstract}
RESUMO
Com o objetivo de investigar características epidemiológicas e clínicas da leishmaniose visceral, em menores de 15 anos, foi realizado um estudo prospectivo em Alagoas no período de 1981 a 1995. Dos 530 casos diagnosticados clinicamente, procedentes em sua maioria da zona rural do Estado de Alagoas, 58\% eram do sexo masculino e $42 \%$ do sexo feminino, sendo 55,3\% abaixo dos 5 anos de idade. As manifestações clínicas mais frequentes na admissão foram: hepatoesplenomegalia, febre e palidez. Amédia de tamanho do fígado e baço dos pacientes com menor tempo de duração da doença ( $<30$ dias) era menor que naqueles doentes há mais tempo ( $\geq 360$ dias). Independente da duração da doença, houve redução do fígado e do baço ao término do tratamento. No entanto, o percentual de redução do baço foi maior nos pacientes que adoeceram há menos tempo. Com relação ao fígado essa diferença não foi observada.
\end{abstract}

Palavras-chaves: Leishmaniose visceral. Alagoas. Epidemiologia. Aspectos clínicos.

\begin{abstract}
In order to investigate epidemiological and clinical characteristics of visceral leishmaniasis in children up to 15 years old, a prospective study was carried out in Alagoas, Brasil from 1981 to 1995. Of the 530 diagnosed cases, predominantly from the rural area of Alagoas State, 58\% were male and $42 \%$ female, being $55.3 \%$ children under 5 years old. The most frequently observed clinical manifestations were: hepatosplenomegaly, fever and parlor. The average size of the liver and the spleen of patients with shorter time of disease ( $<30$ days) were smaller than those presenting sickness for a extended time ( $\geq 360$ days). No matter the length of disease there was reduction of the liver and the spleen after treatment. However, the reduction of the spleen was higher in those patients with less time of sickness. With relation to liver that diference was not observed.
\end{abstract}

Key-words: Visceral leishmaniasis. Alagoas. Epidemiology. Clinical aspects.

A leishmaniose visceral acomete milhares de pessoas no mundo a cada ano, sendo que $90 \%$ delas são oriundas de cinco países, inclusive do Brasil, onde são notificados cerca de 1.980 casos $/ \mathrm{ano}^{9}{ }^{20}$. É uma doença febril de curso prolongado, caracterizada por palidez, emagrecimento, aumento do volume abdominal, hepatoesplenomegalia e edema. Observam-se ainda outras manifestações clínicas como tosse, diarréia, icterícia e sangramentos que dificultam o diagnóstico diferencial com outras doenças, retardando sua identificação, e levando quase sempre 0 paciente à morte quando não tratada ${ }^{19}$.
Os indivíduos infectados podem apresentar desde a forma assintomática, até uma doença de evolução fatal ${ }^{12}$. Segundo diversos autores, o curso clínico da doença pode depender tanto de fatores relacionados à natureza da resposta imune do hospedeiro, à virulência do parasito, como à baixa idade e ao estado nutricional do paciente ${ }^{3}$.

Uma vez que Alagoas é uma região endêmica para a leishmaniose visceral resolveu-se estudar as características da doença nos menores de 15 anos, que correspondem a faixa etária mais freqüentemente acometida no estado.

\footnotetext{
1. Departamento de Clínica Médica do Centro de Ciências da Saúde da Universidade Federal de Alagoas, Maceió, AL. 2. Hospital Escola Dr. Hélvio Auto da Universidade Federal de Alagoas, Maceió, AL. 3. Departamento de Patologia do Centro de Ciências Biológicas da Universidade Federal de Alagoas, Maceió, AL. Endereço para correspondência: Profa Célia M. Pedrosa. R. Papa João Paulo I no 49, Gruta de Lourdes, 57052-130 Maceió, AL, Brasil. e-mail: leishmania@ig.com.br Recebido para publicação 12/12/2002 Aceito em 19/5/2004
} 


\section{MATERIAL E MÉTODOS}

Realizou-se um estudo prospectivo no período de janeiro de 1981 a dezembro de 1995, em pacientes menores de 15 anos admitidos para tratamento de leishmaniose visceral (LV), no Hospital Escola Dr. Hélvio Auto (HEHA), em Maceió-AL( Brasil). 0 Estado de Alagoas está localizado na região centro-oriental do Nordeste brasileiro, e é composto por 101 municípios situados em 3 regiões fisiográficas: leste (litoral e zona da mata), agreste e sertão. 0 HEHA é o único hospital público no Estado responsável pela internação e tratamento de pacientes com doenças infectocontagiosas.

Com 0 objetivo de descrever pacientes com LV, desde a internação até a alta, foi instituído um protocolo para 0 seu acompanhamento. A avaliação dos pacientes, a evolução dos casos, a pesquisa do parasito e 0 preenchimento dos dados eram feitos pelo investigador ou por participantes treinados, sob a supervisão continuada do primeiro. Para minimizar viéses não intencionais, tivemos o cuidado de incluir todos os casos do período de tempo estudado, determinar antecipadamente o ponto final do estudo, e padronizar os métodos de observação?.

Foram incluídos no estudo, pacientes com história e exame físico compatíveis com LV. 0 diagnóstico baseou-se em dados clínicos, como febre prolongada, palidez e hepatomegalia quase sempre associada a esplenomegalia, e epidemiológicos, ou seja, procedência de áreas consideradas endêmicas. Sempre que possível, a confirmação era feita utilizando o exame parasitológico em material obtido através da punção aspirativa da medula óssea. A classificação dos pacientes conforme a duração da doença segue aquela descrita por Kager et al ${ }^{10}$. № momento da admissão foram anotadas em protocolo especíicico, as variáveis biológicas como 0 tamanho do fígado e do baço, ambos medidos na região anterior do abdome abaixo da reborda costal na linha hemiclavicular. 0 tratamento foi realizado segundo 0 protocolo do serviço, inclusive para as intercorrências e patologias associadas, conforme recomendado pela Organização Mundial da Saúde ${ }^{19}$. Aqueles pacientes com icterícia ou que a desenvolveram durante 0 tratamento, não foram tratados com antimonial pentavalente ou este foi suspenso, sendo substituído pela anfotericina B. Durante a evolução, foi observada a data em que a medicação específica teve início e término, e a data em que a febre desapareceu. As complicações e infecções desenvolvidas nesse período, também foram observadas. 0 fígado e 0 baço foram novamente medidos no momento da alta.

Os dados foram analisados com 0 auxílio dos pacotes estatísticos Epi-Info $6.02^{6}$ e SPSS (SPSS for Windows Release 6.0, SPSS Inc. 1989-1993). Foram utilizados testes paramétricos e não paramétricos com intervalo de confiança de $95 \%$ e com nível de significância de 5\% ( p< 0,05).

\section{RESULTAD0S}

Foram estudados 530 pacientes menores de 15 anos, que representavam $82 \%$ do total de pacientes admitidos no HEHA com leishmaniose visceral. Destes, 307 (57,9\%) eram do sexo masculino e $223(42,1 \%)$ do sexo feminino, sendo esta diferença estatisticamente significativa de acordo com a prova de significância de proporção binomial $\left(\chi^{2}=13,0 ; \text { g.l. }=1\right)^{17}$.

A idade dos pacientes variou de seis meses a 14 anos, com média igual a 4,9 $\pm 3,5$ anos. Entre os pacientes do sexo masculino a média de idade foi igual a 5,1 $\pm 3,5$ anos (6 meses a 14 anos e 11 meses), e entre os pacientes do sexo feminino a média de idade foi igual a $4,7 \pm 3,4$ anos ( 10 meses a 14 anos e 11 meses), não sendo essa diferença estatisticamente significativa $(\mathrm{p}=0,11$ g.l. $=1)$. A Tabela 1 mostra a distribuição dos pacientes por sexo e faixa etária.

Tabela 1 - Pacientes internados em Maceió-AL, no período de 1981 a 1995, com leishmaniose visceral, distribuídos por sexo e faixa etária.

\begin{tabular}{|c|c|c|c|c|c|c|c|c|c|}
\hline \multirow{2}{*}{$\begin{array}{l}\text { Idade } \\
\text { (anos) }\end{array}$} & \multicolumn{3}{|c|}{ Pacientes sex $0 \sigma^{2}$} & \multicolumn{3}{|c|}{ Pacientes sexo $\bigcirc$} & \multicolumn{3}{|c|}{ Total } \\
\hline & $\mathrm{n}^{0}$ & $\%$ & $\%$ cum & $\mathrm{n}^{0}$ & $\%$ & $\%$ cum & $\mathrm{n}^{0}$ & $\%$ & $\%$ cum \\
\hline$\overline{<1}$ & 15 & 4,9 & 4,9 & 10 & 4,9 & 4,9 & 25 & 4,7 & 4,7 \\
\hline ||- 5 & 140 & 45,6 & 50,5 & 128 & 57,4 & 61,9 & 268 & 50,6 & 55,3 \\
\hline 10 & 103 & 33,6 & 84,1 & 54 & 24,2 & 86,1 & 157 & 29,6 & 84,9 \\
\hline 10-15 & 49 & 15,9 & 100 & 31 & 13,9 & 100 & 80 & 15,1 & 100,0 \\
\hline Total & 307 & 100,0 & 100,0 & 223 & 100,0 & 100,0 & 530 & 100,0 & 100,0 \\
\hline
\end{tabular}

\% cum - frequêencia cumulativa

Ao longo dos anos verificou-se um maior número de internamentos nos anos de 1986 e 1995, e uma menor freqüência de internamentos em 1981 (Figura 1).

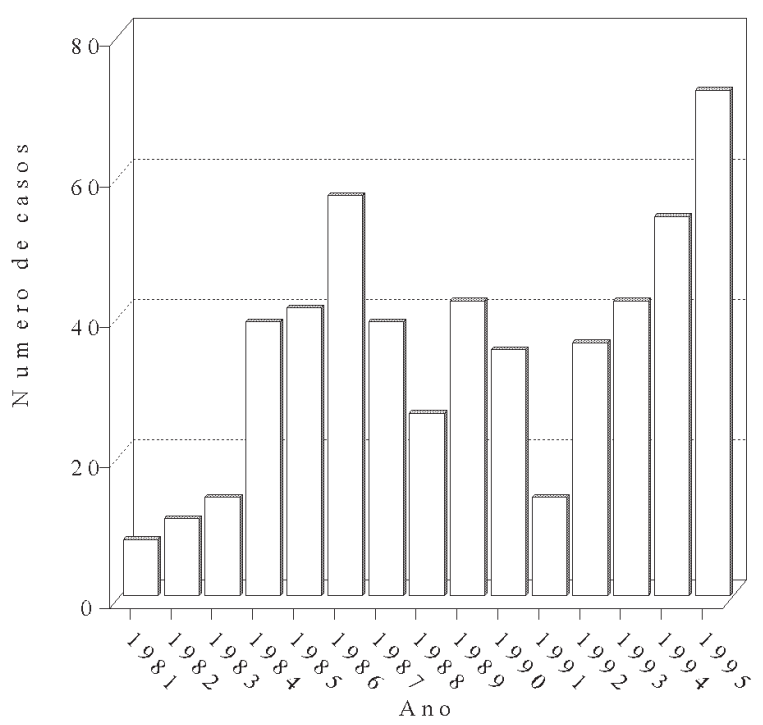

Figura 1 - Distribuição anual de internamentos de pacientes menores de 15 anos com leishmaniose visceral em Maceió-AL, de 1981-1995.

Considerando as regiões fisiográficas do estado, 231 $(43,5 \%)$ dos pacientes procediam do leste alagoano (litoral e zona da mata), 118 (22,2\%) do agreste e 165 (31,3\%) do sertão. Não se conseguiu identificar a região do estado de que eram procedentes 16 (3\%) casos. Os pacientes eram oriundos em sua maioria (77,\%) da zona rural do Estado de Alagoas. 
Dos 530 pacientes admitidos no estudo, 60,5\% deles chegaram ao hospital com até 3 meses de doença (Tabela 2). Em 403 pacientes nos quais foi possível realizar a punção medular, o parasito foi identificado em $81,2 \%$ deles.

Entre as manifestações clínicas mais freqüentemente encontradas na admissão destacaram-se a hepatomegalia, a esplenomegalia, a febre e a palidez (Tabela 3).

Tabela 2 - Duração da doença em pacientes com leishmaniose visceral internados de 1981 a 1995 em Maceió-AL

\begin{tabular}{lccc}
\hline $\begin{array}{l}\text { Duração da } \\
\text { doença (dias) }\end{array}$ & Número de pacientes & Percentagem & $\begin{array}{c}\text { Freqüência } \\
\text { cumulativa }\end{array}$ \\
\hline$<30$ & 119 & 22,4 & 22,4 \\
$30 \mid-90$ & 202 & 38,1 & 60,5 \\
$90 \mid-180$ & 133 & 25,1 & 85,6 \\
$180 \mid-360$ & 47 & 8,9 & 94,5 \\
$\geq 360$ & 29 & 5,5 & 100,0 \\
\hline Total & 530 & 100,0 & 100,0
\end{tabular}

Tabela 3 - Freqüência das manifestações clínicas observadas na internação em pacientes com leishmaniose visceral.

\begin{tabular}{lcc}
\hline Manifestações clínicas & \multicolumn{2}{c}{ Pacientes } \\
\cline { 2 - 3 } & $\mathrm{n}^{\mathbf{0}}$ & $\%$ \\
\hline Hepatomegalia & 530 & 100,0 \\
Palidez & 524 & 98,9 \\
Febre & 513 & 96,8 \\
Esplenomegalia & 503 & 94,9 \\
Aumento dos linfonodos & 456 & 86,0 \\
Cúlios longos & 397 & 74,9 \\
Cabelos secos & 391 & 73,8 \\
Aumento do volume abdominal & 385 & 72,6 \\
Perda de peso & 370 & 69,8 \\
Astenia & 350 & 66,0 \\
Anorexia & 328 & 61,9 \\
\hline
\end{tabular}

0 fígado e 0 baço foram medidos na admissão hospitalar em $530(100 \%)$ e $503(95,5 \%)$ pacientes, respectivamente. As Tabelas 4 e 5 mostram a média do tamanho do fígado e do baço dos pacientes, agrupados de acordo com a duração da doença. Houve aparentemente um aumento gradual desses órgãos que acompanhou 0 tempo de duração da doença. Essas diferenças dos tamanhos médios do fígado e baço mostraram ser estatisticamente significativas ( Análise de variância $\mathrm{p}_{\text {figado }}=0,000078$ e $\mathrm{p}_{\text {baco }}=0,000000$, g.l. $\left.=4\right)$. 0 teste de Diferença Honestamente Significante (DHS) de Tukey, aplicado para localizar esta diferença, revelou que os pacientes com menor tempo de duração da doença ( $<30$ dias) apresentavam 0 tamanho das vísceras menor que aqueles doentes há mais tempo ( $\geq 360$ dias).

Após 0 início do tratamento, naqueles pacientes febris ao internamento, não foi observada diferença estatisticamente significativa entre 0 tempo necessário para a normalização da temperatura e a duração da doença ( $p=0,752$ g.l. $=4)$. Em 267 $(72,1 \%)$ dos pacientes a temperatura se normalizou até 0 70 dia.

Ao término do tratamento o fígado, medido em 420 e 0 baço em 419 pacientes, respectivamente. A média do tamanho do fígado foi igual a $3,5 \pm 2,30 \mathrm{~cm}$ e do baço $4,4 \pm 3,4 \mathrm{~cm}$.

Independente do tempo de duração da doença houve redução do tamanho médio do fígado e do baço após 0 tratamento (Figuras 2A e 2B). A redução percentual desses órgãos ao término do tratamento foi maior naqueles pacientes

Tabela 4 - Tamanho do fígado na admissão hospitalar por duração dedoença em pacientes com leishmaniose visceral em menores de 15 anos.

\begin{tabular}{lcccr}
\hline Duração da & Pacientes & \multicolumn{3}{c}{ Tamanho do fígado $(\mathrm{cm})$} \\
\cline { 3 - 5 } doença & $\mathrm{n}^{\mathbf{0}}$ & média $\pm \mathrm{dp}$ & mínimo & máximo \\
\hline$<30$ & 119 & $4,6 \pm 2,1$ & 0 & 9,5 \\
$30 \mid-90$ & 202 & $5,1 \pm 2,2$ & 0 & 11,0 \\
$90 \mid-180$ & 133 & $5,5 \pm 2,5$ & 0 & 14,0 \\
$180-360$ & 47 & $6,2 \pm 3,1$ & 1,5 & 16,0 \\
$\geq 360$ & 29 & $7,2 \pm 3,8$ & 2,0 & 20,0 \\
\hline Total & 530 & $5,3 \pm 2,5$ & 0 & 20,0 \\
\hline
\end{tabular}

dp - desvio padrão

Tabela 5 - Tamanho do baço na admissão hospitalar por duração de doença em pacientes com leishmaniose visceral em menores de 15 anos.

\begin{tabular}{lcccc}
\hline Duração da & Pacientes & \multicolumn{3}{c}{ Tamanho do baço $(\mathrm{cm})$} \\
\cline { 3 - 5 } doença & $\mathrm{n}^{\mathbf{0}}$ & média $\pm \mathrm{dp}$ & mínimo & máximo \\
\hline$<30$ & 115 & $7,0 \pm 3,9$ & 0 & 21,0 \\
$30 \mid-90$ & 187 & $8,6 \pm 3,6$ & 0 & 20,0 \\
90|- 180 & 127 & $9,7 \pm 3,4$ & 1,0 & 20,0 \\
$180 \mid-360$ & 45 & $10,7 \pm 3,9$ & 0,7 & 20,0 \\
$\geq 360$ & 29 & $12,6 \pm 3,4$ & 5,0 & 18,0 \\
\hline Total & 503 & $9,1 \pm 3,8$ & 0 & 21,0
\end{tabular}

dp: destio padrão

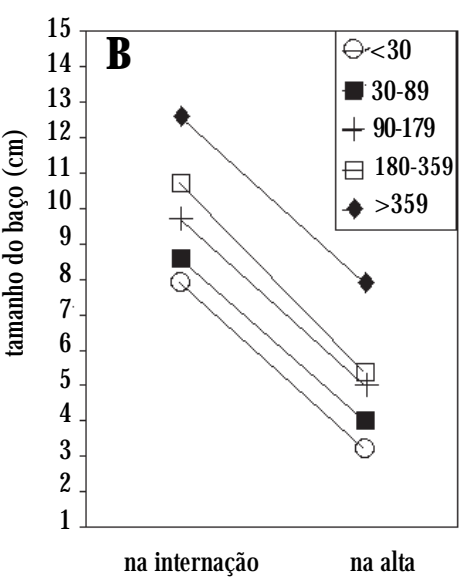

Figura 2 - Média do tamanho do fígado (A) e do baço (B) , na internação e na alta de acordo com a duração da doença. 
com tempo de doença menor (Tabela 6). Considerando a duração da doença, a análise de variância seguido pelo teste de DHS, apontou que o percentual de redução do baço foi maior nos pacientes que adoeceram há menos tempo. Entretanto, com relação ao fígado essa diferença não foi observada. Foram diagnosticadas infecções associadas no momento da internação em 122 (23\%) pacientes, sendo as infecções de vias aéreas em especial a pneumonia (12,1\%) a mais freqüentemente encontrada. Sessenta e sete $(12,6 \%)$ dos pacientes acompanhados faleceram em conseqüência da própria doença ou de alguma complicação.

Tabela 6 - Redução percentual do tamanho médio do baço e do fígado ao término do tratamento.

\begin{tabular}{|c|c|c|c|c|}
\hline \multirow{2}{*}{$\begin{array}{l}\text { Duração da } \\
\text { doença }\end{array}$} & \multicolumn{2}{|c|}{ Baço } & \multicolumn{2}{|c|}{ Fígado } \\
\hline & $\overline{\mathrm{n}^{0} \mathrm{~d}}$ de pacientes & \% de redução* & $\mathrm{n}^{0} \mathbf{0}$ de pacientes & \% de redução* \\
\hline$<30$ & 115 & 63,2 & 119 & 41,3 \\
\hline $30 \mid-90$ & 187 & 53,4 & 202 & 39,2 \\
\hline $90 \mid-180$ & 127 & 48,4 & 133 & 27,2 \\
\hline $180 \mid-360$ & 45 & 49,5 & 47 & 20,9 \\
\hline$\geq 360$ & 29 & 37,3 & 29 & 20,8 \\
\hline
\end{tabular}

* 100 - (média do tamanho do órgão ao término do tratamento x 100) média do tamanho do órgão antes do tratamento

\section{DISCUSSÃ0}

Os primeiros casos que se tem notícia da leishmaniose visceral procedentes de Alagoas datam de 1934, sendo três deles procedentes do litoral e um da zona da mata ${ }^{15}$. Os pacientes incluídos no presente estudo vieram, em ordem crescente de número de casos do agreste, sertão e leste (litoral e zona da mata) do Estado. Portanto, existe uma maior prevalência de casos na região litorânea a exemplo do que ocorre no Nordeste ${ }^{13}$ sendo a procedência uma informação importante para 0 diagnóstico etiológico. Existe uma tendência da leishmaniose visceral de se expandir pelo litoral, como consequêencia da migração de pessoas e seus animais domésticos, possíveis reservatórios da doença, de áreas endêmicas para não endêmicas, causando por vezes surtos, pela presença de susceptíveis. Isso pode ser observado em Japaratinga, cidade situada no litoral Norte de Alagoas, que de 1988 a 1991 notificou dois casos em menores de cinco anos, para em seguida, de 1992 a 1995, notificar 38 casos, dos quais $85 \%$ em menores de cinco anos (CMS Pedrosa: dados não publicados).

A maioria dos pacientes estudados chegou ao hospital entre 30 e 90 dias de doença, achados comparáveis aos de outros autores ${ }^{10}$. É possível que a procura por serviços médicos pelos pacientes e 0 encaminhamento dos mesmos ocorra freqüentemente nesse período, pelo aparecimento de sinais e sintomas sugestivos da doença. A queixa de febre, palidez e de aumento abdominal esteve presente em quase todas as histórias clínicas.

Na admissão chamava à atenção 0 contraste entre os cabelos e os cílios. Enquanto os cabelos eram despigmentados, secos e sem brilho os cilios eram longos e sedosos, alterações semelhantes às referidas por Prata ${ }^{16}$. A esplenomegalia que na casuística de diversos autores ${ }^{41018}$ esteve presente na totalidade dos pacientes, neste estudo estava ausente em $5 \%$ dos casos, sendo esta também a experiência de outros autores que não a encontram em $2 \mathrm{a}$ $18 \%$ dos $\operatorname{casos}^{5}{ }^{21}$. Mesmo sabendo-se que os doentes com IV podem não apresentar todas as manifestações clínicas da doença, a ausência da esplenomegalia sempre dificulta 0 diagnóstico.

De acordo com Marwaha et a $1^{11}$ a duração da doença está patogeneticamente relacionada a esplenomegalia. De fato, os tamanhos do fígado e do baço acompanharam a duração da doença, e quase sempre 0 tamanho do baço predominou sobre 0 do fígado. Apesar disso, a regressão do baço ocorreu mais rapidamente após a instituição do tratamento do que a regressão do fígado, dados estes concordantes com a literatura ${ }^{2} 1014$.

Considerando a ampla distribuição geográfica da LV no Nordeste do Brasil, particularmente no Estado de Alagoas, principalmente em menores de 15 anos, chama-se a atenção dos médicos que clinicam nesta região para a história da doença atual. Se ao exame clínico observa-se palidez e hepatoesplenomegalia, lembrar da possibilidade de leishmaniose visceral. A Organização Mundial de Saúde ${ }^{19}$ adverte que 0 início tardio da terapêutica específica é um fator que piora o prognóstico. Assim, quanto mais precocemente for estabelecido o diagnóstico e instituído 0 tratamento, maior a chance de recuperação dos pacientes.

\section{AGRADECIMENTOS}

Ao corpo clínico e à direção do Hospital Escola Dr. Hélvio Auto.

\section{REFERÊNCIAS BIBLIOGRÁFICAS}

1. Alencar JE. Expansão do calazar no Brasil. Ceará Médico 5:86-102, 1983.

2. Al-Jurayyan AM, Al-Ayed IH, Al-Nasser NS, Al-Mugeiren MA, Boohene AG, Al-Herbish AS. Visceral leishmaniais in infancy and childhood epidemiology and clinicopathological study of 63 cases in Al-Baha Province, Saudi Arabia. Journal of Tropical Pediatrics 38:12-16, 1992.

3. Badaró R, Jones TC, Lorenço R, Cerf BJ, Sampaio D, Carvalho E, Rocha H, Teixeira R, Johnson Jr WD. A prospective study of visceral lesihmaniasis in an endemic area of Brazil. The Journal of Infectious Diseases 154:639649,1986

4. Cascio A, Colomba C, Antinori S, Orobello M, Paterson D, Titone L. Pediatric visceral leishmaniasis in Western Sicily, Italy: a retrospective analysis of 111 cases. European Journal Clinical Microbiology Infectious Disease 21:277-282, 2002.

5. Cole ACE. Kala-azar in East África. Transaction of the Royal Society of Tropical Medicine Hygiene 37:409, 1944.

6. Dean AG, Dean JA, Coulombier D, Brendel KA, Smith DC, Burton AH, Dicker RC, Sullivan K, Fagan RF, Arner TG. Epi Info, version 6.02: a word processing, database, and statistics program for epidemiology on microcomputers. Centers for Disease Control and Prevention. Atlanta, Georgia, USA, 1994.

7. Ebrahim GJ, Sullivan KR. Tipos de Estudos - I. In: Métodos de Pesquisa em Saúde Materno Infantil. Recife, p.89-94, 1996.

8. Grimaldi Jr G, Tesh R. Leishmaniasis of the New World current concepts and implications for future research. Clinical Microbiology Review 6:230-250, 1993.

9. Fundação Nacional de Saúde. Guia de Doenças da Fundação Nacional de Saúde. URL: http://www.funasa.gov.br/guia_epi/htm/doencas/leishmaniose_viceral/ index.htm, 1999-2000. 
10. Kager PA, Rees PH, Manguyu FM, Bhatt KM, Hockmeyer WT, Wellde BT, Lyerly Jr WH. Clinical presentation of visceral leishmaniasis in Kenya: a prospective study of 64 patients. Tropical and Geographical Medicine 35:323-331, 1983

11. Marwaha M, Sarode S, Gupta RK, Garewal G, Dash S. Clinico-hematological characteristics in patients with kala azar. Tropical and Geographical Medicine 43:357-362, 1991.

12. Napier LE. The principles and practice of Tropical Medicine. Mac Millan Company. New York, 1946.

13. Nascimento MDSB, Costa JML, Fiori BIP, Viana GMC, Filho MSG, Alvim AC, Bastos OC, Nakatani M, Reed S, Badaró R, Silva AR, Burattini MN. Aspectos epidemiológicos determinantes na manutenção da leishmaniose visceral no Estado do Maranhão - Brasil. Revista da Sociedade Brasileira de Medicina Tropical 29:233-240,1996.

14. Peña LFL, Yago FC, Amoros EC, Felipe PG, Fabregat RM, Tomas PE. Kalaazar infantil: casuística de una década. Anales Españoles de Pediatria, 39:199-201, 1993
15. Penna HA. Leishmaniose visceral no Brasil. Brasil Médico 46:949950,1934.

16. Prata A. Estudo clínico e laboratorial do calazar. Salvador-BA, Livre Docência - Faculdade de Medicina da Universidade da Bahia, 1957.

17. Snedecor GW, Cochran WG. Statistical Methods. Eighth edition, Iowa State University Press/Ames, 1989.

18. Totan M, Dagdemir A, Muslu A, Albayrak D. Visceral childhood leishmaniasis in Turkey. Acta Paediatrica 91:62-64, 2002.

19. World Health Organization. Report of the informal meeting on the chemotherapy of visceral leishmaniasis. Geneva, 1982.

20. World Health Organization. The leishmaniases and leishmania/HIV coinfections. Fact Sheet № 116, revised May 2000.

21. Zijlstra EE, Siddig AM, El-Hassan AM, El-Toum IA, Satti M, Ghalib HW. Clinical aspects of kala-azar in children from the Sudan: a comparison with the disease in adults. Journal of Tropical Pediatrics 38:17-21, 1992. 